\title{
Pratiques
}

Linguistique, littérature, didactique

\section{Créer le déjà-là, un paradoxe fécond pour penser l'activité scripturale et scientifique de l'auteur de mémoire de master MEEF}

Create the already-there, a fertile paradox to approach the scriptural and scientific activity of the author of a thesis of MEEF Master's degree

\section{Catherine Delarue-Breton}

\section{OpenEdition}

Journals

Édition électronique

URL : http://journals.openedition.org/pratiques/3403

DOI : 10.4000/pratiques.3403

ISSN : 2425-2042

Éditeur

Centre de recherche sur les médiations (CREM)

\section{Référence électronique}

Catherine Delarue-Breton, « Créer le déjà-là, un paradoxe fécond pour penser l'activité scripturale et scientifique de l'auteur de mémoire de master MEEF », Pratiques [En ligne], 173-174 | 2017, mis en ligne le 10 mars 2017, consulté le 01 mai 2019. URL : http://journals.openedition.org/pratiques/3403 ; DOI : 10.4000/pratiques.3403

Ce document a été généré automatiquement le 1 mai 2019.

(c) Tous droits réservés 


\title{
Créer le déjà-là, un paradoxe fécond pour penser l'activité scripturale et scientifique de l'auteur de mémoire de master MEEF
}

\author{
Create the already-there, a fertile paradox to approach the scriptural and \\ scientific activity of the author of a thesis of MEEF Master's degree
}

\section{Catherine Delarue-Breton}

1 La revue Pratiques a consacré plusieurs dossiers à la question complexe de l'écriture dans les apprentissages ou en formation. En ce qui concerne l'université, les notions d'originalité ou de création, centrales dans le présent dossier, sont particulièrement impliquées dans les processus d'écriture des mémoires de master. La présente contribution propose ainsi d'étudier un corpus original de mémoires de master 2 MEEF, élaborés dans le cadre d'un séminaire de recherche relevant de la formation entièrement à distance (FAD).

2 Consciente des difficultés potentielles liées à l'encadrement de mémoire à distance, l'équipe pédagogique a privilégié des choix qui paraissaient susceptibles de faciliter le travail d'acculturation des étudiants à la recherche et au métier d'enseignant. À ce titre, un unique thème de recherche - permettant cependant des questions de recherche différentes - concernant le rôle de la pratique pédagogique dans la compréhension d'un album par les élèves de $\mathrm{CP}$ a été proposé aux étudiants, ainsi qu'un ensemble de textes théoriques sur la question, et un corpus de données constitués d'enregistrements audio de séquences de classes recueillis par l'équipe.

Autrement dit, les étudiants ont travaillé à partir d'un double déjà-là commun : d'une part, un « pré-corpus » de textes scientifiques mettant à leur disposition des savoirs déjà disponibles sur les différentes facettes du thème de recherche concerné, d'autre part un « pré-corpus » de données de terrain. Chacun des deux corpus était en réalité à construire 
par chacun d'eux en fonction de sa question de recherche propre, d'où l'emploi du terme «pré-corpus ». Mais les étudiants ont tous puisé dans ces deux ensembles prédéfinis qui servaient de fonds commun (et les ont éventuellement complétés), ce qui n'est pas habituel dans la démarche de recherche, généralement plus individuelle.

Deux hypothèses principales ont sous-tendu le questionnement de la recherche présentée ici :

- Des mémoires qui se ressemblent formellement peuvent cacher des différences très importantes concernant le travail intellectuel et psychique qui a permis leur élaboration.

- Le travail simultanément scriptural et scientifique qu'implique l'élaboration d'un mémoire de master en formation d'enseignant peut, à certaines conditions, faire évoluer les croyances initiales de leurs auteurs.

5 En effet, si la nécessité d'intégrer la recherche dans la formation des enseignants semble aujourd'hui admise, aux plans institutionnel et scientifique, la visée de celle-ci, et ses effets sont encore aujourd'hui peu connus et insuffisamment décrits. Sans doute, l'impact sur la pratique de classe de l'activité scientifique et scripturale des enseignants en formation - qu'il s'agisse de formation initiale ou continue - peut encore difficilement se mesurer, compte tenu du caractère relativement récent de l'évolution des modalités de formation. L'écriture et la recherche sont en outre susceptibles d'entrainer autant de changements inscrits dans la durée (mais en profondeur) que de changements immédiats (mais plus en surface). Il semble donc particulièrement opportun d'étudier les effets produits sur le rapport au savoir, au métier, au langage et à soi que cette activité est susceptible d'entrainer chez les auteurs de mémoire, au cours de l'élaboration de celui-ci.

Nous évoquerons d'abord les soubassements théoriques de la recherche présentée ici, puis nous décrirons les données analysées. Nous présenterons ensuite plus longuement les résultats de cette analyse, qui concernent d'une part les modes de sélection par les auteurs de mémoire des éléments du déjà-là commun, d'autre part l'usage fait des concepts et leur distribution tout au long du mémoire; nous développerons particulièrement ce dernier point, qui met en évidence le rôle de l'écriture - et plus particulièrement de la textualisation - dans l'élaboration de la pensée propre de l'auteur de mémoire. Nous formulerons enfin quelques remarques sur le rôle de l'activité scripturale et scientifique sur l'évolution des conceptions ou croyances des enseignants.

\section{Appuis théoriques de la recherche}

7 La présence d'un double déjà-là commun nous a permis de nous pencher plus particulièrement sur les usages propres qui en étaient faits par les étudiants, et nous a amenée à repenser les notions de créativité, d'auteur, de textualisation. Mais elle nous a également conduite à prendre en compte la notion de croyance des enseignants, nécessaire pour formaliser les processus psychiques et intellectuels qui sous-tendent l'écriture d'un mémoire de master MEEF. Trois ensembles de notions principales servent ainsi d'appuis théoriques à la recherche que nous présentons ici.

\subsection{Créativité et auctorialité}

Le concept de créativité tel que nous l'entendons s'inspire largement des travaux du psychanalyste anglais D. W. Winnicott (1975), qui considère celle-ci comme une condition 
de l'expérience culturelle, entendue elle-même comme expérience de nouage entre réalité intérieure du sujet et réalité extérieure du monde.

9 Si l'on se place du point de vue de l'expérience universitaire, on peut considérer que celle-ci peut prétendre à l'expérience culturelle lorsqu'elle favorise pour l'étudiant le nouage entre significations internes, déjà élaborées par celui-ci et en lien avec son expérience antérieure, qu'elle soit universitaire ou extra universitaire, et significations externes ou nouvelles, provenant notamment des savoirs théoriques consultés, et qui comprennent une part importante d'altérité. Pour caractériser l'activité de nouage entre réalité psychique intérieure du sujet et apports extérieurs, D. W. Winnicott parle ainsi de créer le déjà-là (ibid., p. 124), activité paradoxale qui suppose, pour l'étudiant, d'élaborer, donc de créer psychiquement lui-même ce qui l'a déjà été ailleurs, par d'autres, et auparavant.

10 Si la créativité, considérée comme un potentiel présent dans chaque individu (Piccardo, 2009), peut être définie comme la capacité à produire du nouveau et s'adapter à un contexte (voir par exemple Lubart, 2003), elle est donc davantage définie par D. W. Winnicott comme une attitude psychique globale vis-à-vis de la réalité extérieure, ce qui en fait dès lors une condition de la capacité à produire, s'adapter ou créer.

11 La question de l'auteur, qui renvoie simultanément à l'idée de production nouvelle, de création originale, mais aussi à celle de promotion ou développement de ce qui est déjà, se pose également en ces termes pour les auteurs de mémoire. Toutefois, le paradoxe pour ces auteurs particuliers est que leur statut se définit à partir d'une double contrainte, qui leur impose à la fois de s'inscrire dans une communauté discursive, et donc de suivre ceux qui les ont précédés, mais dans le même temps de s'en distinguer et s'écarter des sentiers battus par cette communauté pour prétendre y accéder (DelarueBreton, 2014a).

\subsection{Textualisation et dialogisme}

12 La deuxième série de notions qui constituent le soubassement théorique de la recherche concerne l'écriture même du mémoire, et donc la formalisation langagière écrite de la manière dont l'étudiant crée ce déjà-là, autrement dit la textualisation du propos. Le propos en question est cependant lui aussi de nature particulière, puisqu'il s'élabore conjointement à une investigation scientifique, qui ne lui préexiste pas, du moins dans le cas des sciences humaines.

13 L'étude du positionnement énonciatif des auteurs de mémoire, c'est-à-dire de la manière dont ils prennent en charge l'écriture de la recherche, est déterminante pour comprendre comment se construit ce statut. F. Boch (2013), qui s'est intéressée à la production des mémoires de thèse, met en avant trois dimensions significatives pour devenir auteur scientifique : d'une part la manière dont l'auteur prend position dans un texte à visée objectivante, d'autre part la légitimation des enjeux de la recherche, et enfin l'insertion des sources. Dans le cas du mémoire de master, l'étudiant s'approprie des sources en vue de les réinvestir dans une recherche originale, dans un contexte nouveau qui peut parfois s'éloigner beaucoup du contexte de création de ces sources. Le positionnement d'un auteur de mémoire suppose donc à la fois un certain effacement énonciatif et une implication personnelle. Le mode de citation des sources contribue à révéler ce positionnement : F. Boch (2013, p. 559) en distingue trois : reformulation et discours rapporté d'une part (présentes, en ce qui concerne nos mémoires, surtout dans le cadre théorique, 
mais aussi dans l'analyse des données), évocation d'autre part (présente surtout dans l'analyse des données).

14 L'auteur du mémoire peut ainsi être amené à citer des conceptions théoriques auquel il adhère (A. Rabatel [2013], pourrait parler dans ce cas de modalité hétérodialogique de référenciation), mais évoquer également le fait que ces conceptions théoriques le conduisent à revisiter ses propres conceptions antérieures (il s'agirait alors dans ce cas de modalité autodialogique de référenciation). La présence dans les énoncés des mémoires, de propos combinant théorie (donc concepts extérieurs) et conceptions antérieures relèverait dans ce cas d'une double modalité hétéro et autodialogique de référenciation, mais c'est la dimension autodialogique qui retient plus particulièrement notre attention, dans la mesure où elle est susceptible d'attester d'un déplacement de croyance, qui nous intéresse particulièrement dans la formation des enseignants.

\subsection{Croyances des enseignants}

La notion de croyance des enseignants, qui, selon les auteurs, se distingue difficilement de la notion de connaissance, a été étudiée d'une manière approfondie dans la note de synthèse effectuée sur ce thème par M. Crahay et al. (2010). Cette notion nous est d'autant plus utile qu'elle questionne la manière dont s'élaborent chez les enseignants les conceptions du métier en imbriquant les notions sous-jacentes de savoir, doxa, conception, représentation, pour ne citer que quelques-unes d'entre elles.

M. Crahay et al. font le constat qu'il existe un certain consensus sur le fait que les croyances des enseignants évoluent difficilement et résistent aux processus de formation (ibid., p. 106), mais qu'elles sont susceptibles d'évoluer d'autant plus en profondeur qu'elles sont confrontées à la théorie dès la formation initiale (ibid., p. 111). Il nous a donc paru particulièrement intéressant de chercher à comprendre en quoi la confrontation au déjà-là théorique propre à l'élaboration d'un mémoire lors de l'année de master 2 pouvait contribuer à faire évoluer ces croyances, et de repérer les éléments linguistiques présents dans le mémoire susceptibles de l'attester.

\section{Données de la recherche}

17 Nous décrivons ici les données empiriques utilisées par nous dans la recherche présentée dans cette contribution, et les pré-corpus théoriques et empiriques fournis aux auteurs de mémoires pour leur propre recherche.

\subsection{Données de la recherche présentée dans cette contribution}

18 Les données exploitées dans nos travaux pour mieux comprendre l'évolution des enseignants en formation, qui concernent actuellement exclusivement l'enseignement du premier degré, ne se limitent pas à la formation initiale : nous avons analysé également les mémoires d'enseignants déjà en poste (Delarue-Breton, 2014a; Delarue-Breton \& Crinon, 2015). Nous nous en tiendrons cependant pour cette contribution aux mémoires issus de la formation initiale, puisque c'est à ce moment, selon M. Crahay et al. (2010), que l'acculturation théorique semble la plus décisive. Nous avons ainsi étudié 14 documents, que nous désignerons par la suite par des chiffres allant de 1 à 14 (doc.1 à doc.14), et qui correspondent au dernier état du mémoire, celui qui a été soutenu. 


\subsection{Premier pré-corpus fourni aux auteurs de mémoires : le déjà-là théorique}

19 Le pré-corpus de textes théoriques fourni aux étudiants pour les aider dans leur travail était constitué d'une vingtaine de références, concernant les processus d'apprentissage de la lecture (apports de la psychologie cognitive), des apports de la théorie littéraire concernant la notion de compréhension de texte, des apports concernant l'écriture à l'école (approche psychologique, sociologique et génétique des textes) et des apports relevant de la sociologie de l'éducation concernant les inégalités scolaires et les difficultés des élèves en lien avec leur mode de socialisation.

\subsection{Second pré-corpus fourni aux auteurs de mémoires : le déjà-là empirique}

Le pré-corpus de données empiriques fourni aux étudiants était quant à lui constitué de l'observation écologique de 14 séances de classes enregistrées (voir annexe 1), de photographies d'affichages scolaires, de productions d'élèves, et de notes d'observation, recueillies dans un cours préparatoire situé en zone périurbaine classée ZEP à l'époque. Ces séances, toutes centrées sur la lecture d'un album de littérature composite, concernent les différents domaines de la maitrise de la langue tels que définis par le programme officiel : parler, dire, écrire. Elles comportent donc des activités très variées comme le déchiffrage, l'étude des correspondances graphophonémiques, la reconnaissance de mots, la compréhension de texte ou d'image, la production d'écrit, la copie, le débat à visée interprétative, la lecture offerte, etc.

Ce pré-corpus a été complété par des rappels de récit enregistrés par un chercheur de l'équipe, qui a interrogé l'ensemble des élèves tour à tour sur la compréhension de l'album.

Les questions de recherche possibles pour les étudiants pouvaient donc concerner aussi bien la lecture que l'écriture, les interactions langagières, les spécificités du support donné à lire aux élèves, en se focalisant sur les pratiques enseignantes, l'activité de l'élève, les obstacles divers aux apprentissages, etc., en exploitant des données de même type ou en les combinant en fonction de la question de recherche.

\section{Corpus communs, variantes et reprises : des différences dans l'ordre du même}

F. Grossmann (2012), dans un article consacré à la variation dans le discours scientifique, rappelle que c'est le principe même de variation qui est paradoxalement à même de préserver l'unité de la catégorie générique discours scientifique et parle d'une part de diversité pour évoquer les différences entre les disciplines, et d'autre part de variation interne pour évoquer les différences à l'intérieur d'un même champ (ibid., p.141). F. Grossmann ne parle pas de ces documents scientifiques spécifiques que sont les mémoires, et que Y. Reuter (2001) a qualifié d'écrits de recherche en formation. Pour autant, nous pensons que les catégories qu'il propose fonctionnent également pour l'analyse des mémoires, dans la mesure où l'une des caractéristiques communes des 
discours scientifiques réside dans leur visée, qui consiste à entrainer la conviction des lecteurs, opération compliquée dont il rappelle qu'elle ne repose pas seulement sur la clarté scientifique de la démarche ou le statut de la preuve fournie, mais aussi sur des ruptures, des oppositions, des débats (ibid., p. 144). Les différences dont nous allons parler ici relèvent donc de la variation interne, et concernent les constituants communs du discours scientifique (ibid,, p. 145): existence d'un raisonnement, existence d'un dispositif méthodologique, existence d'un système de preuve, existence de résultats. Les axes retenus pour l'analyse des mémoires sont indiqués en annexe 2 .

\subsection{Choix du plan, choix des textes théoriques, choix des données}

24 L'ensemble des documents étudiés laisse apparaitre un plan très comparable d'un auteur à l'autre, qui rappelle la structure du plan IMRad, avec la présence d'une partie concernant le cadre théorique, une partie méthodologie expliquant et justifiant le choix des données, une partie analyse et résultats, l'ensemble étant encadré par une introduction et une conclusion. Au sein de cette structure partagée, enseignée lors des séminaires, on observe cependant des différences majeures, qui attestent déjà d'un positionnement différent des auteurs à l'égard de leurs investigations.

Ainsi, si le plan des documents 4 et 6 laisse bien apparaitre chacune des parties constituantes du mémoire de master susmentionnées, pour autant, les contenus évoqués en 4 traduisent une sélection des références apparemment aléatoire au sein de l'ensemble des sous thèmes abordés en cours (apprentissage de la lecture, compréhension des textes, production d'écrit, construction des inégalités à l'école), et non d'une sélection déterminée par une question de recherche particulière, qui pouvait concerner la compréhension du texte par les élèves ou la production d'écrit par exemple.

À l'opposé, le mémoire 6 atteste d'une sélection des références organisée par une question de recherche spécifique, centrée sur les difficultés de compréhension des élèves en lien avec la transparence des situations didactiques, avec une focalisation particulière sur la dimension complexe de l'implicite dans la lecture (différentes références concernant ce thème ont été fournies par l'auteur du mémoire lui-même).

On retrouve le même type d'opposition entre les mémoires 5 et 10 : le mémoire 5 sélectionne des références en fonction de la question de recherche, tandis que le mémoire 10 , comme le mémoire 4 , intègre toute sorte de références sur la lecture, l'écriture, etc. On note cependant une différence de positionnement entre les deux auteurs du mémoire 10 et du mémoire $4:$ tandis que l'auteur 4 ne formule pas vraiment de question de recherche précise dans le mémoire, et parle d'une manière générale de difficultés de lecture et d'écriture d'élèves de milieux défavorisés, le mémoire 10 formule au contraire une question de recherche spécifique, concernant l'influence de la pratique enseignante sur la compréhension de l'album concerné, question de recherche qui laisse donc de côté le thème de l'écriture. Pourquoi, dans ce cas, en avoir conservé les références dans le cadre théorique?

Notre interprétation est la suivante (voir notamment Delarue-Breton, 2014a) : le cursus de master MEEF place les étudiants dans une triple posture susceptible d'être conflictuelle si elle n'est pas élaborée donc travaillée en formation (mais susceptible d'être porteuse si elle l'est), qui les conduit simultanément à restituer du savoir (posture d'étudiant), à diffuser du savoir (posture d'enseignant), et à produire du savoir (posture de chercheur). 
29 Ainsi, tout en respectant en surface les attendus formels du genre discursif scientifique (il existe un raisonnement, un dispositif méthodologique, un apparent système de preuve et des analyses sinon des résultats), l'auteur du mémoire 4 semble ne pas s'être positionné clairement comme apprenti chercheur, mais comme étudiant qui restitue des savoirs, ce qu'il était probablement jusqu'alors. Et en effet, les savoirs ici restitués le sont convenablement, ils sont articulés entre eux et pour autant qu'on puisse en juger, ils sont compris.

30 En revanche, l'auteur du mémoire 10, qui semble pénétrer plus avant les tenants et aboutissants du genre mémoire de master MEEF (Delarue-Breton, 2014b), mais qui ne le maitrise pas encore tout à fait, restitue à la fois des savoirs utiles pour sa recherche, et d'autres savoirs, qui lui seront certes utiles en tant qu'enseignant (posture de transmission de savoir) mais pas dans le mémoire.

31 Pour ce qui est du mémoire 5, son auteur, comme dans le cas du mémoire 6, rassemble effectivement des références articulées par une question de recherche spécifique, ayant trait aux effets de la lecture oralisée sur la compréhension des élèves : de même que dans le cas du mémoire 6, l'inventivité de la question de recherche participe selon nous de l'attitude créative de son auteur, qui a construit la question à partir des deux pré-corpus qui forment le double déjà-là commun aux étudiants, et non restitué des savoirs sur un thème donné.

32 Pour ce qui est du choix des données, nous pouvons formuler des remarques comparables : chaque auteur a opéré une sélection au sein du pré-corpus de données de terrain, mais tandis que certains d'entre eux ont effectué des choix guidés exclusivement par leur question de recherche, et intégrant le caractère innovant ou renouvelant de celle-ci, d'autres ont sélectionné des données concernant aussi bien la lecture ou la compréhension de texte, que l'écriture, ce qui donne une apparence de cohérence à l'ensemble puisque le cadre théorique (qui mentionne des références dans ces deux domaines) coïncide, dans une certaine mesure, au corpus de données, mais en réalité ne participe pas à l'analyse créative de celles-ci. Les données sélectionnées dans ce genre de cas correspondent à un thème de recherche, mais pas à une question de recherche.

Ainsi, dans le mémoire 8, la recherche envisagée porte sur l'écriture, comme l'indique le titre. Pourtant, la sélection des données élimine, sans justification explicite, certaines des productions d'écrit réalisées par les élèves au cours de la séquence, et à l'opposé, retient l'ensemble des rappels de récit effectués par les chercheurs afin d'augmenter le matériau linguistique permettant l'analyse de la compréhension du support par les élèves. Par ailleurs, alors que 9 séances sur 14 comprennent des activités d'écriture (séances 1, 4, 6, 7, $8,9,10,11$ et 14), seule la séance 11 a été retenue, choix qui est justifié dans le mémoire non pas parce qu'elle comprend une production d'écrit particulière, mais parce qu'elle porte sur un passage de l'album déterminant en termes de difficulté de compréhension. Les productions écrites réalisées par les élèves au cours de cette séance sont d'ailleurs finalement exclues de l'analyse. Les données retenues pour ce mémoire sont ainsi des données concernant au moins autant la compréhension de texte que l'écriture, de même que les choix théoriques effectués.

À l'opposé, le mémoire 6, dont la question de recherche concerne spécifiquement les difficultés de compréhension en lecture des élèves, et qui accorde une large place, nous l'avons dit, à la dimension de l'implicite dans l'album exploité, et à la transparence des enjeux didactiques dans la séance, sélectionne des données très ciblées. L'auteur prélève 
ainsi d'abord, au sein de chacune des 14 séances, les bribes d'enregistrements concernant les activités dédiées exclusivement à la compréhension de l'album par les élèves, en laissant de côté les autres (comme les activités de déchiffrage ou de travail des correspondances graphophonémiques) ; il sélectionne ensuite les rappels de récit, qui lui permettront d'évaluer la compréhension du récit par chaque élève, créant ainsi un corpus original à partir d'un déjà-là commun, où aucune séance n'est utilisée entièrement, mais aucune non plus laissée entièrement de côté.

\subsection{Usages des concepts et distribution tout au long du mémoire : textualisation et dialogisme}

Nous ne pouvons dans le cadre de cet article donner le détail des différentes formes de textualisation rencontrées ; nous nous bornerons donc à en donner à voir les modalités les plus marquantes, et qui semblent révéler le plus l'activité dialogique des auteurs, autrement dit la manière dont le texte du mémoire dialogue avec les textes des auteurs du cadre théorique.

En poursuivant notre étude du mémoire 6 , nous découvrons ainsi une forme de textualisation qui exploite les facilités offertes par l'écriture numérique, qui consiste à insérer des tableaux dans le corps du mémoire, et à sélectionner certains des items mentionnés dans les cases du tableau en en grisant le texte.

Voici un extrait de l'un de ces tableaux, qui occupe dans son entier deux pages dans le mémoire (doc. 6, p. 36-37) :

Tableau 1.

\begin{tabular}{|c|c|c|}
\hline Séances & Déroulement & Enjeu supposé de l'activité \\
\hline 8 & 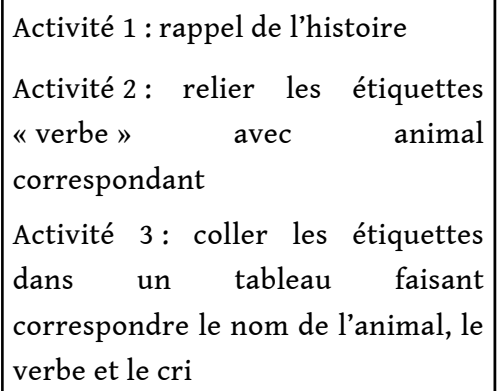 & $\begin{array}{l}\text { Se remémorer l'histoire, avoir une idée globale } \\
\text { être capable de la restituer, placer les élèves en } \\
\text { situation d'interprète (Cèbe \& Goigoux, 2014) }\end{array}$ \\
\hline 9 & $\begin{array}{l}\text { Activité } 1 \text { : lecture offerte, arrivée } \\
\text { de l'ours } \\
\text { Activité 2: imaginer la suite de } \\
\text { l'histoire à l'oral } \\
\begin{array}{l}\text { Activité } 3: \text { écrire la suite de } \\
\text { l'histoire }\end{array}\end{array}$ & $\begin{array}{l}\text { Ancrer l'élève dans un questionnement en } \\
\text { s'appuyant sur le texte. Entrer dans le processus } \\
\text { de compréhension en s'appuyant sur le texte } \\
\text { (Tauveron, 2002). } \\
\text { Comprendre les inférences causales, les états } \\
\text { mentaux des personnages (Cèbe \& Goigoux } \\
2014 \text { ) }\end{array}$ \\
\hline
\end{tabular}

Dans cet exemple, nous interprétons l'usage du tableau et le surlignage en gris comme permettant de mettre en avant d'une manière efficace et rapide pour le lecteur les choix opérés par l'auteur. Ainsi, l'ensemble des 14 séances sont représentées dans le tableau, 
ainsi que 29 des activités qui les constituent. Par ailleurs, la mise en regard dans le tableau des activités sélectionnées avec des références théoriques permet un rapprochement économique car peu couteux en espace, au sein d'un mémoire de format court (100 000 signes environ). Le tableau rend ainsi visible l'activité intellectuelle et psychique de l'auteur, qui progresse de tableaux en tableaux entrecoupés de textes, évoluant dans sa réflexion selon un schéma "en entonnoir", où le tableau qui suit reprend les items sélectionnés au sein du précédent, et les développe à partir de catégories d'analyse plus fines inspirées par le cadre théorique. Le tableau suivant reprend ainsi exclusivement les enjeux correspondant aux activités grisées dans le tableau 1, puis les met en regard avec les propos de l'enseignante s'y rapportant et leur perception par les élèves.

Voici un extrait du tableau suivant, qui occupe, dans son entier, trois pages dans le mémoire (doc. 6, p. 39-41) :

Tableau 2.

\begin{tabular}{|c|c|c|}
\hline Enjeux supposés & $\begin{array}{l}\text { Termes de l'enseignante } \\
\text { exprimant les enjeux }\end{array}$ & $\begin{array}{l}\text { Compréhension de l'enjeu et réalisation } \\
\text { par les élèves }\end{array}$ \\
\hline $\begin{array}{l}\text { S.3/ Activité 1: } \\
\text { Se remémorer } \\
\text { l'histoire, la } \\
\text { restituer, avoir } \\
\text { une idée globale. } \\
\text { Minutes } 1 \text { à } 7\end{array}$ & $\begin{array}{l}\text { Consigne "Je vais donc } \\
\text { demander à un élève dans la } \\
\text { classe de me dire ce que l'on sait } \\
\text { déjà sur l'histoire du Bonnet } \\
\text { Rouge.»min.1. } \\
\text { "Qu'est-ce qu'on sait déjà? ». } \\
\text { «Alors on ne parle pas de la } \\
\text { couverture, on parle de l'histoire. } \\
\text { Qu'est-ce qu'on sait déjà de } \\
\text { l'histoire? min. } 2 \\
\text { Enjeux exprimés: } \\
\text { remémorer l'histoire, la } \\
\text { restituer } \\
\text { Enjeux non exprimés: avoir } \\
\text { une idée globale. }\end{array}$ & $\begin{array}{l}\text { Pour certains, l'histoire est comprise et } \\
\text { restituée: "Chapeau rouge, il part sur le } \\
\text { chemin. Et soudain, un arbre, une branche. } \\
\text { Bonnet Rouge, rien, il continue le chemin" } \\
\text { min. } 3^{1} \\
\text { Pour d'autre, seuls des éléments de } \\
\text { l'histoire ont été compris, compréhension } \\
\text { en ilots: "c'est l'histoire de quoi?" "d'un } \\
\text { lutin» min. } 1 \\
\text { Pour d'autres l'histoire n'est pas du tout } \\
\text { comprise: "Chapeau Rouge va chez sa } \\
\text { mamie» min. } 3 \text {, "Pour lui apporter quelque } \\
\text { chose» min. } 4\end{array}$ \\
\hline
\end{tabular}

Les modes de citation relèvent dans les deux tableaux de l'évocation; mais dans le premier cas, il s'agit d'une forme d'évocation que nous qualifions d'explicite (les références sont mentionnées), tandis que nous la qualifions d'implicite dans le second, ce qui peut attester d'une forme d'appropriation créative du déjà-là théorique. Ainsi, la référence à $\mathrm{S}$. Cèbe et R. Goigoux, par exemple, dans le tableau 1 (ligne 2, colonne 3), n'apparait plus dans le tableau 2 (ligne 2, colonne 1); en revanche, la colonne 2 du tableau 2 utilise les contenus théoriques pour distinguer les enjeux exprimés des enjeux non exprimés, apportant ainsi une part de créativité dans l'analyse. L'imbrication du déjà-là théorique et des données se poursuit ainsi tout au long du mémoire, à travers des sélections successives au sein des tableaux, entrecoupés de textes de synthèse qui répondent en partie à la question de recherche. En voici un exemple :

Parmi les 11 séances de cette séquence que j'ai analysées, j'ai noté que les enjeux de

l'activité sont rarement énoncés. [...] Par exemple, dans la première séance, 
l'enseignante explique aux élèves qu'ils vont devoir observer la couverture, et dire à quoi cela leur fait penser, ce qui représente la tâche à effectuer. En revanche, rien n'exprime en quoi cela va les aider à entrer dans le récit et à en comprendre le sens, donc l'enjeu d'apprentissage de l'activité reste implicite. La plupart du temps, les élèves réalisent la tâche, mais on s'aperçoit au moment où chacun doit raconter l'album, que malgré tout, une bonne partie de la classe n'a pas saisi le sens du récit et donc que les activités menées n'ont pas permis d'entrer dans la compréhension.

[Doc. 6, p. 38, souligné par nous.]

41 Si le propos est sans doute un peu radical, il traduit cependant une appropriation intellectuelle des concepts convoqués dans le cadre théorique, qui se réinvestissent ailleurs, et donc d'hétérodialogisme implicite. Mais dans le cas présent, on note également une forme d'autodialogisme attesté selon nous par la formule malgré tout, qui évoque certes l'idée d'une réserve que l'on pourrait traduire ainsi : * "en dépit des efforts consacrés par l'enseignante, une bonne partie de la classe n'a pas saisi le sens... », mais aussi sans doute une forme de concession théorique de l'auteur du mémoire. Celle-ci pourrait se traduire ainsi * « il faut bien reconnaître qu'une bonne partie de la classe n'a pas saisi le sens... ", résultat qui est apparu lors de l'analyse des rappels de récit, et qui vient illustrer les thèses de coconstruction des inégalités évoquées dans le cadre théorique.

En termes de textualisation, il est intéressant de noter ici que ce paragraphe, qui s'insère entre les tableaux 1 et 2 situés pages 36-37 et 39-51, s'appuie pourtant aussi sur une analyse des rappels de récit («au moment où les élèves doivent raconter l'histoire ») qui n'apparait que bien plus tard dans le mémoire (pages 52 à 55).

43 Aussi, si la disposition des tableaux rend bien compte de la forme en entonnoir - donc linéaire, dans une certaine mesure - de l'analyse, les paragraphes rédigés mettent plutôt en évidence le caractère récursif de l'élaboration des résultats, qui laisse penser que l'écriture même du texte a une fonction de rassemblement des différents éléments connus au moment de la rédaction, attestant ainsi au plan énonciatif d'une implication du sujet qui devient partie prenante au plan théorique.

Manifester une attitude créative, c'est ainsi d'abord s'autoriser une sélection propre - et par conséquent appropriée parce que déterminée par un questionnement personnel - et faire dialoguer cette situation avec une autre, autrement dit effectuer des rapprochements d'univers distincts entre eux. Mais il nous apparait aussi à travers cet exemple que c'est également produire un univers de pensée qui contribue à l'unification de l'expérience de l'auteur du mémoire, en favorisant le nouage des significations entre réalité intérieure et extérieure.

45 Si un tiers des mémoires étudiés repose ainsi sur un questionnement central qui tient ensemble les différents constituants du mémoire évoqués par F. Grossmann, d'autres sont traversés par des questions successives ou par une interrogation globale qui ne permettent pas d'unifier l'ensemble, et donc ne se textualisent pas de manière homogène.

Le mémoire 13 par exemple, ne choisit pas parmi les pistes de recherche proposées par l'équipe pédagogique, et tente de prendre en charge l'ensemble des questions possibles: la recherche envisagée concerne aussi bien la lecture que l'écriture, elle s'intéresse aussi bien aux pratiques enseignantes qu'à l'activité des élèves, à l'oral comme à l'écrit, et elle envisage de questionner également la complexité du support, dans une approche plus successive qu'orchestrée.

Le mémoire 8, dont nous avons déjà fait mention, montre quant à lui un glissement d'un thème à l'autre, à l'insu de l'auteur, semble-t-il : 
Au regard de ces théories, nous pouvons nous demander dans le cadre de notre recherche comment les élèves apprennent-ils [sic] à écrire au CP à partir de supports complexes. Plus particulièrement, nous pourrions nous interroger sur les pratiques enseignantes en ce qu'elles pourraient favoriser ou induire des écarts de compréhension d'un album composite chez des élèves de milieu défavorisé. En quoi les pratiques enseignantes peuvent-elles induire une mauvaise compréhension de l'album ? [Doc. 8, p. 12-13, souligné par nous.]

La question de recherche porte explicitement dans la première phrase sur le thème de l'écriture, conformément au titre du mémoire (le choix de ce thème est d'ailleurs affirmé dès la page 1 , puis confirmé page 8 et page 11). Puis, il s'agit d'évaluer les pratiques enseignantes sur le thème de la compréhension, au cours des deux phrases suivantes. En termes de textualisation, on note que la troisième phrase reprend en interrogation directe la phrase précédente, qui relève de l'interrogation indirecte. Cette reprise selon la modalité directe vient résumer d'une manière incisive la phrase précédente, mais contribue peut-être ainsi à figer le questionnement sur l'un de ses aspects latéraux, au détriment du questionnement initialement envisagé.

On aurait donc affaire ici à une sorte d'écrit de travail, qui progresse à travers l'écriture elle-même du mémoire, mais sans unification de l'expérience scripturale sous-tendue par un questionnement propre, et sans enjeu clairement identifié de la recherche. On note en effet la phrase suivante, à la fin de l'introduction: « Notre recherche a un réel intérêt dans la mesure où les questions d'écriture en cycle 2, et notamment pour les élèves de $\mathrm{CP}$, ont été jusqu'ici peu explorées ». Ce propos, qui correspond sans doute à un désir de se conformer aux attendus du genre, vient légitimer une question qui ne sera finalement pas traitée dans le mémoire, qui porte plutôt sur la compréhension de texte.

Ce cas de figure, qui atteste d'un glissement de la question de recherche initiale vers un autre thème à l'insu de l'auteur n'est pas exceptionnel : on retrouve le même phénomène dans le mémoire 3, qui portera in fine lui aussi sur la compréhension de texte, mais qui annonce également un travail de recherche sur la production d'écrit (malgré un titre centré cette fois sur la compréhension):

Notre recherche, ancrée dans la didactique du français, cherche à analyser dans quelles mesures les pratiques d'enseignement de la lecture et de l'écriture influencent-elles la production d'écrits des élèves? Nous nous demanderons plus particulièrement dans quelles mesures l'utilisation d'un support comme l'album qui peut être perçu comme complexe dans un milieu défavorisé - peut-être un vecteur à ses productions d'écrits. [Doc. 3, p. 10.]

51 Dans ce cas de figure, les données sélectionnées, contrairement au mémoire 8, sont des productions écrites d'élèves. L'une des clefs permettant de comprendre le glissement réside selon nous dans la définition du cadre d'analyse des données: "Les productions des élèves seront donc analysées du point de vue de la compréhension » [Doc. 3, p. 23.]

Il n'est donc pas prévu qu'elles soient analysées du point de vue de la production, mais de la réception du support exploité, ce qui semble traduire une certaine confusion entre entrée dans l'écriture et entrée dans l'écrit. Nous trouvons confirmation de cette analyse dans le cadre théorique, qui atteste de cette confusion :

L'écriture n'est pas une activité nouvelle pour l'élève qui entre en CP. Il a eu l'occasion en maternelle de côtoyer le monde de l'écrit à travers la découverte d'album de littérature de jeunesse, l'écriture de son prénom mais également par la dictée à l'adulte. [Doc. 3, p. 6, souligné par nous.] 
53 L'instabilité de la question de recherche de ces deux mémoires, sans doute produite, au moins dans le deuxième cas, par une confusion ou incertitude théorique, entraine des hésitations dans l'ensemble du mémoire: ainsi, l'analyse des productions écrites du mémoire 3 se fait comme annoncé dans le cadre d'analyse du point de vue de la compréhension du support, mais envisage néanmoins des facteurs orthographiques, ce qui nous conduit à considérer que l'analyse ne s'est pas non plus vraiment stabilisée sur la compréhension.

Lorsque les mémoires ne sont pas traversés par un questionnement propre, original, ceux-ci se voient ainsi tenus plutôt par une structure extérieure qui reprend les constituants évoqués par F. Grossmann (2012), qui font figure de cases à remplir ou d'items à renseigner.

Inversement, lorsque les mémoires sont traversés par un questionnement propre stabilisé, fut-il évolutif et ramifié, le caractère spécifique de la question permet de tenir ensemble, de l'intérieur, les choix théoriques et empiriques. Cette implication du sujet auteur dans le mémoire se traduit souvent paradoxalement par des hésitations énonciatives qui opèrent à son insu. On note ainsi dans le mémoire 12 , dont le questionnement concerne le rôle spécifique du débat interprétatif dans la compréhension de l'album par les élèves, une hésitation entre l'emploi du nous et du je :

Nous allons donc voir quels sont les éléments qui ont été retenus par les chercheurs comme pouvant déterminer la complexité d'un album... Ces différents éléments vont donc m'aider à déterminer ce qui fait la complexité du Bonnet rouge. [Doc. 12, p. 5-6, souligné par nous.]

Cette hésitation énonciative nous apparait, dans ce cas précis, révélatrice d'un moment particulier de l'implication du sujet dans la recherche en cours, et donc comme un marqueur linguistique possible du fait de devenir auteur.

Pour synthétiser notre propos concernant les différentes formes de textualisation rencontrées, qui tantôt oscillent entre maitrise des concepts articulés à l'analyse, et glissements $d u$ questionnement à l'insu même de leurs auteurs, nous pouvons mentionner le fait que les mémoires qui attestent d'un mouvement de pensée créatif à partir du double déjà-là commun sont ceux qui présentent un triple dialogisme, et non seulement l'un ou l'autre :

- Le premier consiste à faire dialoguer efficacement les auteurs sources entre eux.

- Le deuxième consiste en un dialogue entre l'auteur du mémoire et les auteurs sources.

- Le troisième, que nous illustrons ci-dessous, et qui participe de l'évolution des croyances des enseignants, consiste en un dialogue entre l'auteur du mémoire et lui-même, autrement dit en un dialogue de soi à soi.

\subsection{De l'évolution des croyances des enseignants en formation}

L'évolution des croyances des enseignants, dont nous rappelons qu'elle est peu aisée, mais d'autant plus envisageable que les croyances sont confrontées d'entrée de jeu à des concepts théoriques, nous parait pouvoir être saisie dans le mémoire à travers différents types d'énoncés : propos explicites des auteurs, souvent en introduction ou en conclusion du mémoire, mentionnant des changements de conception, déplacements de la question de recherche, modifications dans la sélection des données, quand elles correspondent (c'est-à-dire dans la plupart des cas) à des découvertes de l'auteur sur le métier, sur les élèves, sur la discipline, etc., ou encore présentation des résultats. 
59 Ainsi le mémoire 5 , très abouti et dynamique, portant sur la lecture théâtralisée comme moyen susceptible - ou non - de favoriser la compréhension du texte par les élèves (le pré-corpus fourni aux étudiants a été ici complété par un autre recueil de données effectué par l'auteur du mémoire), formule ainsi en conclusion un commentaire sur les acquis de cette expérience scientifique :

Enfin notre connaissance très parcellaire des différents acquis en sciences de l'éducation nous a-t-elle permis de créer tous les liens nécessaires à l'étayage de notre analyse?

Je terminerais ce mémoire sur une réflexion métacognitive en choisissant de m'interroger sur le transfert des connaissances acquises au cours de cette approche de la recherche en sciences de l'éducation. [Doc. 5, p. 46, souligné par nous.]

On retrouve ici la rupture énonciative (passage du nous au je) évoquée précédemment, qui porte sur un retour sur l'expérience vécue, et plus particulièrement sur la réflexion théorique, ainsi que sur les effets produits sur le sujet auteur par cette expérience. Cette rupture s'accompagne d'un redoublement du dire («transfert des connaissances acquises $»)$, un peu surprenant compte tenu de la grande qualité linguistique de l'ensemble du mémoire. L'apparition de ce double phénomène linguistique inattendu nous conduit à l'interpréter comme la marque d'un mouvement important dans les conceptions de l'auteur du mémoire, sur lequel il souhaite s'expliquer. La suite du texte, qui revient sur l'un des auteurs cités dans le cadre théorique, évoque en effet le rôle du développement «de compétences stratégiques nécessaires à la compréhension des implicites qui dominent la littérature de jeunesse aujourd'hui» (Doc.5, p.59), attestant ainsi simultanément d'une découverte des caractéristiques de la littérature de jeunesse actuelle, et de la nécessité de leur prise en compte au plan didactique. L'auteur évoque ensuite, dans un propos qui clôt le mémoire, le protocole mis en place, en se positionnant non plus comme chercheur mais comme professionnel, quittant ainsi la posture de chercheur producteur de savoir pour celle d'enseignant transmetteur de savoir :

C'est en croisant les apports des différents chercheurs du monde de l'éducation sur un thème bien précis, que j'ai tenté de concevoir une pratique pédagogique me permettant d'exploiter au mieux les compétences que j'ai pu développer au cours d'une vie professionnelle relativement éparse. [Doc. 5, p. 59.]

61 Toujours en conclusion, l'auteur du mémoire 11, qui n'évoque cependant pas explicitement dans le mémoire des changements de conception ou une réflexion métacognitive, propose un renouvèlement de la réflexion engagée :

Il est très intéressant d'observer en milieu scolaire l'impact de la mise en place de telle ou telle approche didactique sur la compréhension de l'élève. Nous avons vu les difficultés rencontrées par l'enseignant lors de la mise en place de l'étude d'un album composite de littérature de jeunesse. L'enseignant en essayant de mettre en place une séquence aidant la compréhension de l'album a parfois induit les élèves dans la construction de fausses représentations (en séance 9 surtout), ce qui a mis en difficulté de nombreux élèves. Il serait intéressant de poursuivre ce travail de recherche autour du rôle et de la responsabilité de l'enseignant dans la mise en place de séquence de compréhension en lecture. [Doc. 13, p. 42, souligné par nous.]

Dans ce passage, l'emploi à deux reprises de la formule «il est/serait intéressant » avec variation temporo-modale, invite à penser que les découvertes effectuées par l'auteur lors de l'analyse des données de terrain le conduit à revisiter ses conceptions concernant le rôle de l'enseignant, généralement considéré comme évidemment favorable par les étudiants au début de leur formation. Dès lors, le thème de la réflexion, qui a porté dans 
le mémoire - et d'une manière particulièrement impliquée et dynamique - sur l'impact effectif des images dans la compréhension du texte par les élèves, se déplacerait vers la question du rôle et de la responsabilité de l'enseignant, question certainement sensible pour l'auteur, lauréat du concours et qui devient donc lui-même enseignant à l'issue du master.

\section{Conclusion} qu'elle le parait au premier abord, et qu'elle peut parfois même s'avérer chaotique. De ce point de vue, elle reflète assez bien, nous semble-t-il, les mouvements de pensée de leurs auteurs, et les cheminements complexes qui font le propre de l'activité scripturale et scientifique. Pour autant, toutes les progressions ne sont pas du même ordre : que peuton dire des processus de textualisation observés?

Tout d'abord, que textualisation et unification de l'expérience ont partie liée : lorsque l'appropriation des concepts est créative au sens winiccottien, et qu'elle vient renouveler le rapport au monde, elle sert aussi le projet de recherche, et les différentes rubriques du mémoire convergent, ce qui n'est pas le cas quand elle a une simple visée de restitution de savoir.

Certains mémoires, qui ne sont pas structurés par une question de recherche authentique (c'est-à-dire nouvelle, produite et investie par son auteur), semblent ainsi constitués de textes successifs plutôt que de parties d'un même texte. Ils peuvent par exemple montrer de grandes qualités théoriques (concepts compris et clairement reformulés, dialogue efficace entre les auteurs, etc.), sans que les concepts convoqués constituent l'armature conceptuelle de la recherche conduite. Autrement dit, la partie théorique du mémoire, qui fait texte en soi, ne constitue pas un cadre théorique du mémoire pour autant.

Les deux questions que nous posions en introduction nous semblent ainsi étroitement liées: les processus de textualisation sont déterminés par l'usage du déjà-là, autrement dit par le renouvèlement apporté par l'auteur du mémoire, renouvèlement qui s'enracine d'abord dans l'originalité et la pertinence du questionnement. Ce caractère central du questionnement nous conduit à penser que les inégalités d'accès au savoir qui caractérisent aussi bien les étudiants que les élèves, gagneraient à être pensées aussi en termes d'inégalité d'accès au questionnement.

La question de savoir pour qui les auteurs de mémoires créent ce déjà-là est cependant également à considérer: pour eux-mêmes, d'abord, puisqu'il s'agit de nouer des significations nouvelles avec des significations antérieures; mais aussi pour la communauté scientifique et professionnelle, ce qui gagnerait à être mieux pris en compte. Certains mémoires proposent en effet des questionnements renouvelés, des rapprochements théoriques audacieux mais convaincants, ou des méthodologies efficaces, et sont insuffisamment valorisés actuellement. 


\section{BIBLIOGRAPHIE}

восн, F. (2013). « Former les doctorants à l'écriture de la thèse en exploitant les études descriptives de l'écrit scientifique ». Linguagem em (Dis)curso, Tubarao, SC 13-3, p. 543-568.

CRAHAY, M., WANLIN, P., ISSAIEVA, É. \& LADURON, I. (2010). « Fonction, structuration et évolution des croyances (et connaissances) des enseignant ». Revue française de pédagogie 172, p. 85-129.

DELARUE-BRETON, C. (2014a). «Créativité, au(c)torisation et dialogisme : le mémoire de master MEEF, miroir de l'expérience psychique ?» SHS Web of Conferences 8, p. 2751-2766.

- (2014b). « Le mémoire de master MEEF : un nouveau genre universitaire ? » Diversité 177, p. $50-55$.

DELARUE-BRETON, C. \& CRINON, J. (2015). « De l'usage des concepts dans les mémoires de master Métiers de l'enseignement ». Le Français aujourd'hui 188, p. 79-88.

GROSSMANN, F. (2012). « Pourquoi et comment cela change ? standardisation et variation dans le champ des discours scientifiques ». Pratiques 153/154, p. 141-160.

LUBART, T. (2003). Psychologie de la créativité. Paris : A. Colin.

PICCARDO, E. (2009). «Introduction ». Synergies Europe 4, p. 7-12.

RABATEL, A. (2013). « Positions, positionnements et postures de l'énonciateurs ». Linha d'Agua 26-2, p. 159-183.

REUTER, Y. (2001). « Je suis comme un autrui qui doute. Le discours des autres dans l'écrit de recherche en formation ». Lidil 24, p. 13-27.

WinNicotT, D. W. (1975) [1971]. Jeu et réalité. L'espace potentiel. Trad. de l'anglais par C. Monod et J.B. Pontalis. Paris : Gallimard.

\section{ANNEXES}

\section{Annexe 1. Séquence « Bonnet Rouge » (description succincte) - classe de CP}

Wenninger, B. \& Rowe, J.-A (2000). Le Bonnet rouge. Paris : Nord-Sud.

Résumé de l'album : un lutin pourvu d'un bonnet rouge se promène dans les bois, et se fait prendre son bonnet par une branche à son insu. Une grenouille, une souris, un lapin, un hérisson, un oiseau, un renard, un sanglier, un loup et un ours pénètrent alors successivement dans le bonnet tombé par terre. Puis vient une puce, qui demande à entrer elle aussi, mais qui fait fuir l'ensemble des animaux présents. La puce s'installe 
alors seule dans le bonnet, qui sera retrouvé in fine par le lutin, qui le replacera sur sa tête.

\section{Séance 1}

- Tâche 1 : présentation de l'album par l'enseignante

- Tâche 2 : observation libre de la page de couverture par les élèves

- Tâche 3: description orale collective des objets figurant sur l'image (animaux notamment)

- Tâche 4: production d'un dessin individuel par les élèves, représentant ce qu'ils pensent que le livre raconte

- Tâche 5 : travail d'écriture : les élèves sont invités à dire ce qu'ils voient (Sur l'image, je vois...), en s'aidant d'un abécédaire pour trouver les lettres nécessaires

- Tâche 6 : les élèves sont invités à lire individuellement les mots figurant au tableau, et à les recopier sur un document ad hoc sous l'image correspondante, afin de compléter leur dictionnaire de mots.

\section{Séance 2}

- Tâche 1: repérage des mots connus à partir de l'incipit de l'album recopié en cursives au tableau

- Tâche 2 : lecture collective à voix haute du texte

- Tâche 3 : repérage de sons : les élèves sont invités à entourer les sons [in] [b] et [s] (sic) au tableau

\section{Séance 3}

- Tâche 1 : rappel du texte lu la veille par les élèves, puis lecture par l'enseignante

- Tâche 2 : rappel par les élèves des noms d'animaux qui entrent dans le bonnet, en suivant l'ordre d'arrivée

- Tâche 3 : découpage et lecture d'étiquettes portant les noms des animaux présents dans l'album

- Tâche 4 : à partir de l'énoncé du nom de l'animal par l'enseignante, les élèves sont invités à retrouver l'étiquette correspondante

- Tâche 5 : mise en ordre des étiquettes représentant les animaux en fonction de leur arrivée dans l'histoire

\section{Séance 4}

- Tâche 1 : lecture collective à voix haute de syllabes

- Tâche 2 : scansion orale collective des syllabes des noms d'animaux

- Tâche 3 : copie par les élèves du nom de deux animaux sur l'ardoise, et écriture d'un nom d'animal imaginaire constitué d'une syllabe de chacun des deux noms d'animaux copiés

- Tâche 4: sur un document fourni, copie des deux noms d'animaux, et du nom de l'animal composite, puis rédaction d'une phrase indiquant le lieu de vie (au choix des élèves) de l'animal imaginaire (Il vit...)

\section{Séance 5}

- Tâche 1 : rappel de l'histoire ; les élèves sont invités à placer dans un bonnet tenu par l'enseignante les images des animaux nommés

- Tâche 2 : échanges concernant la taille des animaux (peuvent-ils vraiment tenir dans le bonnet ?), puis concernant l'activité d'écriture (écrire, c'est mettre un titre, faire des phrases, etc.) 
- Tâche 3 : lecture magistrale du texte, que les élèves ont sous les yeux; repérage individuel des structures répétitives (les élèves doivent colorier en rouge ce qui se répète)

- Tâche 4 : mise en commun

\section{Séance 6}

- Tâche 1 : retour collectif sur la structure répétitive de l'histoire

- Tâche 2: découpage d'étiquettes mots d'une phrase, puis identification des étiquettes correspondant aux mots énoncés par l'enseignante

- Tâche 3 : reconstitution individuelle de la phrase par les élèves, avec les étiquettes

- Tâche 4 : les élèves sont invités à compléter en écriture cursive un texte à trou, qui reprend la même phrase

\section{Séance 7}

- Tâche 1: à partir d'un abécédaire de noms d'animaux affiché dans la classe, les élèves sont invités à choisir des noms de cinq animaux autres que ceux de l'album

- Tâche 2 : copie de la liste d'animaux choisis sur le document fourni

- Tâche 3: rédaction d'une phrase sur le document fourni en ordonnant les noms d'animaux par ordre de taille (Dans mon bonnet il y a...)

\section{Séance 8}

- Tâche 1 : lecture à voix haute par les élèves d'étiquettes indiquant le nom du cri d'un animal, attribution du cri à l'animal correspondant, mention du verbe désignant ce cri

- Tâche 2 : écriture du nom de l'animal et du nom du cri correspondant

\section{Séance 9}

- Tâche 1 : lecture magistrale d'un passage de l'album

- Tâche 2 : émission d'hypothèses collectives orales sur la suite du passage lu

- Tâche 3 : rédaction de la suite hypothétique du passage (en autonomie ou en dictée à l'adulte)

\section{Séance 10}

- Tâche 1 : encodage de mots (à l'écrit) avec le son [b] : arbre - poubelle - corbeau boire - robinet - bobine - bras - balançoire - cartable - biberon

\section{Séance 11}

- Tâche 1 : description collective orale de l'une des images de l'album projetée

- Tâche 2 : lecture individuelle du texte correspondant à l'image, copié au tableau en écriture cursive

- Tâche 3 : identification et entourage du mot «puce » dans ce texte

- Tâche 4 : débat interprétatif à partir des questions suivantes: la puce veut-elle entrer dans le bonnet? Que répondent les animaux ? Pourquoi ? Est-ce que c'est un problème de place ? Pourquoi lui disent-ils non? Quel est le problème?

- Tâche 5 : production d'écrit : les élèves sont invités à rédiger la suite de l'histoire ( $L a$ puce va...)

\section{Séance 12}

- Tâche 1 : lecture magistrale de l'album dans son entier

\section{Séance 13}

- Tâche 1 : étude collective d'une production d'élève de la séance 9 (tâche 3) recopiée au tableau (commentaires, propositions d'amélioration) 


\section{Séance 14}

- Tâche 1 : correction de l'exercice sur le son [b] (séance 10)

- Tâche 2 : encodage des mots à l'écrit

\section{Annexe 2. Axes de l'analyse des mémoires}

\section{Organisation du sommaire}

- Plan type (introduction, cadre théorique, méthodologie, analyse, résultats, conclusion)

- Plan thématisé

Évolution de la question de recherche et problématisation, ramification du questionnement

- En amont du cadre théorique

- Au sein du cadre théorique

- Au cours de l'analyse

- Lors de la présentation des résultats

- Synthétisée ou reformulée en conclusion

\section{Traitement des conceptions initiales}

- Présence vs absence de conceptions initiales

- Mention des conceptions tout au long du mémoire: maintien/reconfiguration/ reformulation

- Émission d'hypothèses, prédictions

Choix des références à partir du pré-corpus théorique fourni

- Choix aléatoire

- Choix ciblé en fonction de la question de recherche

Présence et usage des références théoriques citées en bibliographie

Usage des références conceptuelles du cadre théorique

- Dans l'analyse des données

- Ailleurs dans le mémoire (conclusion notamment)

Présence dans le mémoire de références théoriques autres que celles mentionnées dans le cadre théorique

- Recours à d'autres références lors de l'analyse

- Recours à d'autre références lors de la présentation des résultats

- Recours à d'autres références en conclusion

\section{Choix des données}

- Choix aléatoire parmi les données du pré-corpus de séances

- Choix ciblé parmi les données du pré-corpus de séances en fonction de la question de recherche

- Ajout de données originales

Textualisation du propos, choix énonciatifs, modalisations diverses

- Au sein des différentes rubriques du mémoire 
- Entre les rubriques du mémoire

\section{Formes du dialogisme}

- Présence d'un dialogue entre auteurs sources

- Présence d'un dialogue entre l'auteur du mémoire et les auteurs sources

- Présence d'un dialogue entre l'auteur du mémoire et lui-même (de soi à soi)

\section{Questionnement scientifique/questionnement professionnel}

- Présence d'un rapprochement entre questionnement scientifique et professionnel

- Absence de rapprochement entre questionnement scientifique et professionnel

- Confusion entre questionnement scientifique et professionnel

\section{NOTES}

1. L'élève explique que le lutin, qui porte un bonnet rouge (et qu'il désigne tantôt sous le nom de Chapeau rouge, tantôt sous le nom de Bonnet rouge), perd son bonnet attrapé par une branche lors d'une promenade en forêt, mais ne s'en aperçoit pas (« rien ») et continue son chemin.

\section{RÉSUMÉS}

Les étudiants qui se destinent aux métiers de l'enseignement doivent produire un mémoire de master, dont le genre relève à la fois du scientifique, pour ce qui est du cadre théorique et de l'analyse des données, et du professionnel pour ce qui est de l'objet de recherche. Le cadre théorique de ce mémoire, autrement dit son soubassement conceptuel, est constitué d'énoncés qui puisent chez d'autres auteurs, et à ce titre participe d'une forme particulière de déjà-là dans l'écriture. Mais par ailleurs, le corpus étudié, qui peut être constitué de propos enregistrés en classe, de paroles d'acteurs, constitue également une autre forme de déjà-là, qui doit être pris en charge dans le mémoire. L'écriture de ce mémoire convoque donc plusieurs niveaux de déjà-là, qui font autant de plans du discours qu'il convient de textualiser. La présente contribution se propose, à partir de l'étude de 14 mémoires achevés de master MEEF, de se pencher plus particulièrement sur les questions suivantes: Quel usage est fait du déjà-là? Comment caractériser le nouveau dans l'usage du déjà-là? De quoi est fait le nouveau de la pensée d'un auteur apprenti chercheur? Quelles marques linguistiques permettent d'affirmer que se développe la pensée propre d'un auteur, qui cite et reformule autrui? Dans quelle mesure l'ajustement des matériaux linguistiques «fait texte", autrement dit où commence la textualisation? Peut-on affirmer que le document produit ne fait parfois pas "texte ", et n'est constitué que d'une juxtaposition d'énoncés? L'analyse présentée montre que ces 14 mémoires, qui ont été élaborés à partir de cadres théoriques comparables et ont analysé des données identiques, sont pourtant constitués de textes fort différents, aux plans simultanément conceptuel, scriptural et scientifique.

The students who plan to work in teaching professions have to write a thesis of Master's degree, which is both scientific, as for the theoretical frame and the data analysis, and professional as for the object of search. The theoretical frame of this thesis, that is its abstract base, consists of statements which have their source at other authors, and as such participates of a particular 
shape of already-there in the writing. But besides, the studied corpus which can gather discussions recorded in class, actors' words, also represents another shape of already-there, which must be taken care of within the thesis. Thus, the writing of this thesis convenes several levels of already-there, which make so many various speeches that writers should textualize. With references to 14 theses of MEEF Master's degree, this paper suggests dealing more particularly with the following issues: Which use is made of the already-there? How to characterize the new in the use of the already-there? What's the new made of in the thought of an author who is a researcher apprentice? Which linguistic markers enable to assert that the proper thought of an author has been developing, when this author quotes and reformulates others? - To what extent does the adjustment of linguistic materials "make/create a text", in other words where does textualization begin? Can we assert that the document which is produced doesn't sometimes "make text", and consists only in a juxtaposition of statements? This analysis shows that these 14 theses, which were developed from comparable conceptual frameworks and analyzed the same data, are however made up of very different texts, simultaneously to conceptual, scriptural and scientific plans.

\section{INDEX}

Mots-clés : écriture scientifique, recherche et formation, textualisation, conceptions

Keywords : scientific writing, research and training, textualization, belief

\section{AUTEUR}

\section{CATHERINE DELARUE-BRETON}

Université Paris Est, EA 4384 CIRCEFT-Escol, Université Paris 8, UPEC, F-94010, France 